\title{
EDITORIAL
}

\section{Developing a new generation of breast cancer clinical gene expression tests}

\author{
Zuzana Kos $^{1}$ and Torsten O Nielsen ${ }^{2^{*}}$ \\ See related research by Martin et al., http://breast-cancer-research.com/content/16/2/R38
}

\begin{abstract}
When treatment decisions are based purely on clinicopathological factors, many women with estrogen receptor-positive/human epidermal growth factor receptor 2-negative cancers are overtreated. Gene expression profiles are valuable clinical tools that stratify the recurrence risk to identify patients most likely to benefit from adjuvant systemic therapies. Building upon greater understanding of tumor biology and more rigorous approaches to validation (including independent studies with a high level of evidence), several second-generation multigene tests have been developed. In the previous issue, Martin and colleagues report the third clinical validation study for EndoPredict, a distributed assay to assess risk of distant recurrences in estrogen receptor-positive/human epidermal growth factor receptor 2-negative women. The authors confirm the assay's independent prognostic value in premenopausal and postmenopausal, node-positive women treated with contemporary chemotherapy followed by endocrine therapy. EndoPredict did not, however, predict benefit from adding paclitaxel. Predictive signatures for selecting among chemotherapy regimens remain an area needing further development.
\end{abstract}

When treatment is based solely on clinicopathological risk factors, many women with estrogen receptor-positive/ human epidermal growth factor receptor 2-negative $\left(\mathrm{ER}^{+} / \mathrm{HER} 2^{-}\right)$tumors are overtreated - subjected to morbidity from cytotoxic chemotherapy for negligible benefit. Identifying patients safely treated by endocrine

\footnotetext{
* Correspondence: torsten@mail.ubc.ca

${ }^{2}$ Genetic Pathology Evaluation Centre, Department of Pathology and Laboratory Medicine, University of British Columbia, 2660 Oak Street, Vancouver, BC V6H 3Z6, Canada

Full list of author information is available at the end of the article
}

therapy alone has driven the development of prognostic gene expression assays.

In the previous issue, Martin and colleagues describe the third clinical validation of EndoPredict (EP; Sividon Diagnostics GmbH, Cologne, Germany) [1], a secondgeneration multigene test trained to predict distant recurrence in $\mathrm{ER}^{+} / \mathrm{HER} 2^{-}$tumors, and by extension the need for adjuvant chemotherapy. EP was previously validated in prospective-retrospective analyses of endocrine-treated postmenopausal $\mathrm{ER}^{+} / \mathrm{HER} 2^{-}$breast cancer patients in two clinical trials (ABCSG-6 and ABCSG-8) [2].

Genomic-based assays developed from complex, highdimensional data are susceptible to overfitting. Clinical validation must be performed in entirely independent datasets using predefined, locked-down classifier algorithms and analysis plans. Martin and colleagues' study exemplifies the rigor required by Simon and colleagues for a formal prospective-retrospective study to contribute to generating level IB evidence [3]. The authors present $\mathrm{EP}$ validation results in $\mathrm{ER}^{+} / \mathrm{HER} 2^{-}$patients from the GEICAM/9906 clinical trial of node-positive women treated with contemporary chemotherapy. The prognostic ability of EP remains robust in this higher risk group, identifying a 10 -year metastatic rate of $7 \%$ in the predefined EP low-risk group (versus $30 \%$ in the high-risk group). Including tumor size and nodal status as the EPclin classifier identifies a small (13\%) but impressively low-risk cohort of women who experienced no distant recurrences at 10 years. As the whole of this cohort received chemotherapy, the clinical utility of this finding (to avoid chemotherapy) is difficult to infer, although the $100 \%$ metastasis-free survival in both the fluorouracil-epirubicin-cyclophosphamide and the fluorouracil-epirubicin-cyclophosphamide-paclitaxel randomized arms does imply no benefit from adding paclitaxel.

The authors speculate that the EPclin low-risk group (recurrence-free at 10 years) in this 5-year endocrine therapy-treated population may identify women not needing extended endocrine therapy, consistent with the 
Table 1 Overview of selected multigene signatures for breast cancer

\begin{tabular}{|c|c|c|c|c|c|c|c|c|c|c|}
\hline \multirow{2}{*}{$\begin{array}{l}\text { Assay; } \\
\text { platform, } \\
\text { clinical } \\
\text { material }\end{array}$} & \multirow[t]{2}{*}{ Training parameter } & \multirow{2}{*}{$\begin{array}{l}\text { Approval or } \\
\text { endorsement }\end{array}$} & \multirow{2}{*}{$\begin{array}{l}\text { Analytical } \\
\text { validity: } \\
\text { published } \\
\text { assay } \\
\text { validation }\end{array}$} & \multirow{2}{*}{$\begin{array}{l}\text { Clinical } \\
\text { validity: } \\
\text { prognosis } \\
\text { validation }\end{array}$} & \multicolumn{5}{|c|}{ Predicting treatment benefit using randomized clinical trials } & \multirow{2}{*}{$\begin{array}{l}\text { Randomized } \\
\text { prospective } \\
\text { trials }\end{array}$} \\
\hline & & & & & Tamoxifen & Herceptin & $\begin{array}{l}\text { Chemotherapy } \\
\text { versus no } \\
\text { chemotherapy }\end{array}$ & $\begin{array}{l}\text { Specific } \\
\text { agent: } \\
\text { anthracycline }\end{array}$ & $\begin{array}{l}\text { Specific agent: } \\
\text { taxane }\end{array}$ & \\
\hline $\begin{array}{l}\text { Breast Cancer } \\
\text { Index; RT- } \\
\text { PCR, FFPE } \\
\text { (central) }\end{array}$ & $\begin{array}{l}\text { Outcome }\left(\mathrm{ER}^{+}, \mathrm{pNO},\right. \\
\text { endocrine-treated women) } \\
\text { MGI component - biology } \\
\text { (tumor grade related genes) } \\
\text { H:I component - outcome } \\
\text { (recurrence in tamoxifen- } \\
\text { treated women) }\end{array}$ & No & No & $\begin{array}{l}\text { ATAC [13], } \\
\text { Stockholm [17], } \\
\text { multiple } \\
\text { nonrandomized } \\
\text { trial cohorts }\end{array}$ & $\mathrm{No}^{\mathrm{a}}$ & No & No & No & No & No \\
\hline $\begin{array}{l}\text { EndoPredict; } \\
\text { RT-PCR, FFPE } \\
\text { (distributed) }\end{array}$ & $\begin{array}{l}\text { Outcome (distant } \\
\text { recurrence in endocrine- } \\
\text { treated } \mathrm{ER}^{+} / \mathrm{HER}^{-} \mathrm{pNO} / \mathrm{pN}^{+} \\
\text {women) }\end{array}$ & CE Mark & Yes $[18,19]$ & $\begin{array}{l}\text { ABCSG6 [2], } \\
\text { ABCSG8 [2], } \\
\text { GEICAM/9906 } \\
\text { [1] }\end{array}$ & No & No & No & No & $\begin{array}{l}\text { GEICAM/9906 [1] } \\
\text { (failed to predict } \\
\text { benefit) }\end{array}$ & No \\
\hline $\begin{array}{l}\text { IHC4; IHC, } \\
\text { FFPE } \\
\text { (distributed) }\end{array}$ & $\begin{array}{l}\text { Outcome (distant } \\
\text { recurrence in } E R^{+} \\
\text {endocrine-treated women) }\end{array}$ & No & No & $\begin{array}{l}\text { ATAC [14], } \\
\text { TEAM [20] }\end{array}$ & No & No & No & No & No & No \\
\hline $\begin{array}{l}\text { MammaPrint; } \\
\text { microarray, } \\
\text { fresh and } \\
\text { FFPE (central) }\end{array}$ & $\begin{array}{l}\text { Outcome (5-year metastasis } \\
\text { rate in pNO women) }\end{array}$ & $\begin{array}{l}\text { FDA (fresh): risk for } \\
\text { distant metastasis, } \\
<61 \text { years, stage I and } \\
\text { II, tumor } \leq 5 \mathrm{~cm} \text { and } \\
\text { node-negative }\end{array}$ & No & $\begin{array}{l}\text { Multiple } \\
\text { nonrandomized } \\
\text { trial cohorts } \\
\text { including } \\
\text { RASTER }\end{array}$ & No & No & No & No & No & $\begin{array}{l}\text { MINDACT } \\
\text { prognosis } \\
\text { validation (to } \\
\text { report 2015) }\end{array}$ \\
\hline $\begin{array}{l}\text { Mammostrat; } \\
\text { IHC, FFPE } \\
\text { (central) }\end{array}$ & $\begin{array}{l}\text { Outcome (unselected } \\
\text { cohort of breast cancer } \\
\text { patients) }\end{array}$ & No & No & $\begin{array}{l}\text { NSABP-B14, } \\
\text { NSABP-B20 [15] } \\
\text { multiple non- } \\
\text { randomized trial } \\
\text { cohorts }\end{array}$ & No & No & $\begin{array}{l}\text { NSABP-B20 } \\
( \pm \text { CMF) [15] (all } \\
\text { women benefit } \\
\text { - high risk } \\
\text { benefit the } \\
\text { most) }\end{array}$ & No & No & No \\
\hline $\begin{array}{l}\text { Oncotype DX; } \\
\text { RT-PCR, FFPE } \\
\text { (central) }\end{array}$ & $\begin{array}{l}\text { Outcome (recurrence in } \\
\text { mainly tamoxifen-treated } \\
\mathrm{ER}^{+}, \mathrm{pNO} \text { women) }\end{array}$ & $\begin{array}{l}\text { NCCN, ASCO, St. } \\
\text { Gallen (role for } \\
\text { identifying women } \\
\text { that may benefit from } \\
\text { chemotherapy) }\end{array}$ & Yes [21] & $\begin{array}{l}\text { NSABP-B14 [9], } \\
\text { NSABP-B28 [22], } \\
\text { SWOG8814 [23], } \\
\text { multiple non- } \\
\text { randomized trial } \\
\text { cohorts }\end{array}$ & $\begin{array}{l}\text { NSABP-B14 } \\
\text { [24] (largest } \\
\text { benefit in } \\
\text { quantitative } \\
\text { ER high/ } \\
\text { recurrence } \\
\text { risk low } \\
\text { patients) }\end{array}$ & No & $\begin{array}{l}\text { NSABP-B20 } \\
( \pm \text { CMF) [25], } \\
\text { SWOG8814 } \\
\text { ( } \pm \text { CAF) [23] } \\
\text { (benefit from } \\
\text { chemotherapy } \\
\text { with high } \\
\text { recurrence score) }\end{array}$ & No & $\begin{array}{l}\text { NSABP-B28 [22] } \\
\text { (failed to predict } \\
\text { a benefit) }\end{array}$ & $\begin{array}{l}\text { TAlLORx } \\
\text { (node- } \\
\text { negative, to } \\
\text { report 2015) } \\
\text { RxPONDER } \\
\text { (one to three } \\
\text { positive nodes, } \\
\text { recruiting) }\end{array}$ \\
\hline $\begin{array}{l}\text { PAM50 } \\
\text { (research } \\
\text { based assay); } \\
\text { RT-PCR and } \\
\text { microarray, } \\
\text { FFPE and } \\
\text { fresh } \\
\text { (distributed) }\end{array}$ & $\begin{array}{l}\text { Biology (identification of } \\
\text { major molecular subtypes) }\end{array}$ & N/A research assay & No & $\begin{array}{l}\text { NCIC-MA5 [26], } \\
\text { NCIC-MA12 [27], } \\
\text { GEICAM/9906 } \\
\text { [28], multiple } \\
\text { nonrandomized } \\
\text { trial cohorts }\end{array}$ & $\begin{array}{l}\text { NCIC-MA12 } \\
\text { [27] (luminal } \\
\text { subtype } \\
\text { predicts } \\
\text { benefit) }\end{array}$ & $\begin{array}{l}\mathrm{NOAH} \\
\text { [29] } \\
\text { (HER2- } \\
\text { enriched } \\
\text { benefits } \\
\text { the most) }\end{array}$ & No & $\begin{array}{l}\text { NCIC-MA5 } \\
\text { [26] (CMF vs. } \\
\text { CEF; } \\
\text { epirubicin } \\
\text { benefit in } \\
\text { HER2- } \\
\text { enriched } \\
\text { subtype only) }\end{array}$ & $\begin{array}{l}\text { GEICAM/9906, } \\
\text { CALGB/9342 and } \\
\text { CALGB/9840 [30] } \\
\text { (low proliferation } \\
\text { score predicts } \\
\text { weekly paclitaxel } \\
\text { benefit) }\end{array}$ & No \\
\hline
\end{tabular}


Table 1 Overview of selected multigene signatures for breast cancer (Continued)

\begin{tabular}{|c|c|c|c|c|c|c|c|c|c|c|}
\hline $\begin{array}{l}\text { Prosigna; } \\
\text { nCounter, } \\
\text { FFPE } \\
\text { (distributed) }\end{array}$ & $\begin{array}{l}\text { Biology (subtype); outcome } \\
\text { (ROR score) }\end{array}$ & $\begin{array}{l}\text { CE Mark, Health } \\
\text { Canada, FDA: } \\
\text { prediction of } 10 \text {-year } \\
\text { DRFS in ER+, node } 0 \\
\text { to } 3 \text {, postmenopausal } \\
\text { women treated with } \\
\text { endocrine therapy }\end{array}$ & Yes [31] & $\begin{array}{l}\text { ATAC [32], } \\
\text { ABCSG08 [33] }\end{array}$ & No & No & No & No & No & $\begin{array}{l}\text { RXPONDER } \\
\text { (one to three } \\
\text { nodes, } \\
\text { recruiting; } \\
\text { embedded } \\
\text { additional } \\
\text { analysis) }\end{array}$ \\
\hline
\end{tabular}

CAF, cyclophosphamide, doxorubicin, fluorouracil; CEF, cyclophosphamide, epirubicin, fluorouracil; CMF, cyclophosphamide, methotrexate and fluorouracil; DRFS, distant relapse-free survival; ER, estrogen receptor; FDA, US Food and Drug Administration; FFPE, formalin-fixed paraffin-embedded; HER2, human epidermal growth factor receptor 2; H:I, HOXB13:IL17BR; IHC, immunohistochemistry; MGI, molecular grade index; N/A, not applicable; pNO, pathological lymph node-negative; $\mathrm{pN}^{+}$, pathological lymph node-positive; ROR, risk of recurrence; RT-PCR, reverse transcription polymerase chain reaction. Breast Cancer Index: bioTheranostics, San Diego, CA, USA; EndoPredict: Sividon Diagnostics GmbH, Cologne, Germany; IHC4: MammaPrint: Agendia, Amsterdam, The Netherlands; Mammostrat: Clarient, Inc., Aliso Viejo, CA, USA; Oncotype: Genomic Health, Redwood City, CA, USA; PAM50: NanoString Technologies Inc., Seatlle, WA, USA; Prosigna: NanoString Technologies Inc., Seattle, WA, USA. ${ }^{a}$ Nested cohort study using material from NCIC CTG MA.17 - HOXB13/L17BR predictive of benefit from extended letrozole. 
ABCSG-8 trial [4]. This promising idea must be interpreted cautiously given that only 16 of the 74 EPclin low-risk patients had 10-year follow-up data. Both the aTTom and ATLAS trials have shown that survival benefits of extended hormonal therapy become more apparent after year $10[5,6]$.

Several multigene prognostic assays have now been developed for use in $\mathrm{ER}^{+} / \mathrm{HER} 2^{-}$breast cancers. Firstgeneration assays including MammaPrint (MammaPrint: Agendia, Amsterdam, The Netherlands) and Oncotype DX (Oncotype: Genomic Health, Redwood City, CA, USA) suffered from early methodological issues, most seriously a failure to maintain rigorous separation between training and validation sets, and inclusion of nonluminal and/or HER2 $^{+}$tumors in their training sets, thereby allowing these high-risk tumors to skew outcome-related gene selection away from the relevant patient group [7-9]. MammaPrint was specifically trained around early relapse (within 5 years) in node-negative women, most having received no adjuvant systemic therapy, and has not been shown to predict late recurrence outside the original training-validation cohort. Oncotype DX heavily weighed the tamoxifen-only arm of the NSABP-B20 trial in its training set, where most recurrences occurred within 5 years, and has diminished prognostic ability beyond year 5 [10].

More recently, building upon biological and technical advances and more rigorous approaches to validation, second-generation multigene tests have been developed, including the Breast Cancer Index (BCI: bioTheranostics, San Diego, CA, USA), PAM50 (PAM50: NanoString Technologies Inc., Seattle, WA, USA) and EP. The Breast Cancer Index combines a molecular grade index (quantifying tumor grade-associated genes) and a two-gene ratio, HOXB13:IL17BR, related to estrogen signaling [11]. PAM50, unlike signatures trained around outcome, was developed as a biological classifier of the major intrinsic molecular subtypes of breast cancer [12]. These three assays predict both early and late recurrences $[4,10,13]$.

IHC4 and Mammostrat (Mammostrat: Clarient, Inc., Aliso Viejo, CA, USA) immunohistochemical panels are also prognostic in early breast cancer $[14,15]$. IHC4 uses standard pathology markers (ER, progesterone receptor, HER2 and Ki67) to provide prognostic information comparable with Oncotype DX [14]. Immunohistochemical staining and scoring does suffer from limited analytical reproducibility, probably contributing to Martin and colleagues' identification of low Ki67 scores $(<14 \%$; a published cutoff point for good-prognosis luminal A tumors) in a surprisingly high fraction (almost three-quarters) of this node-positive cohort [16].

Each of these gene expression and immunohistochemical panels identifies a good prognosis group that may not need chemotherapy. Emerging evidence suggests that some panels identify women at such low risk of late recurrence that they may safely avoid extended endocrine therapy. For high-risk women, however, the question is not one of chemotherapy versus no chemotherapy, but rather a question of which chemotherapy agent(s) will be most effective for which patients - a true predictive indication. In Martin and colleagues' report, the EP score did not predict benefit from adding weekly paclitaxel to fluorouracil-epirubicincyclophosphamide chemotherapy. Outcome-trained signatures from nonchemotherapy populations are unlikely to predict between chemotherapy regimens; Table 1 summarizes some relevant features of the referenced molecular signatures, including predictive studies.

What does the future hold for gene expression signatures? Cheaper and faster next-generation sequencing has been touted as the pinnacle of personalized medicine, destined to render multigene expression assays obsolete. However, the genetic complexity of tumors (copy number variations, chromosome-scale structural changes, thousands of mutations, epigenetic changes and intratumoral genetic heterogeneity) is proving even more complex than anticipated. Much as the increased detail from electron microscopy never did replace light microscopy for cancer diagnosis, the broader signatures detected by representative gene expression profile assays, reflecting clinically significant patterns common across many patients, are likely to remain relevant for important treatment decisions.

\section{Abbreviations \\ EP: EndoPredict; ER: Estrogen receptor; HER2: Human epidermal growth factor receptor 2 .}

\section{Competing interests}

TON reports a proprietary interest in the PAM50 assay, which has been licensed to Nanostring Technologies. ZK has no competing interests.

\section{Author details}

${ }^{1}$ Department of Laboratory Medicine and Pathobiology, University of Toronto, 1 King's College Circle, Toronto, ON M5S 1A8, Canada. ${ }^{2}$ Genetic Pathology Evaluation Centre, Department of Pathology and Laboratory Medicine, University of British Columbia, 2660 Oak Street, Vancouver, BC V6H 3Z6, Canada.

\section{Published: 07 Jul 2014}

\section{References}

1. Martin M, Brase JC, Calvo L, Krappmann K, Ruiz-Borrego M, Fisch K, Ruiz A, Weber KE, Munarriz B, Petry C, Rodriguez CA, Kronenwett R, Crespo C, Alba E, Carrasco E, Casas M, Caballero R, Rodriguez-Lescure A: Clinical validation of the EndoPredict test in node-positive chemotherapy-treated $\mathrm{ER}^{+} / \mathrm{HER2}{ }^{-}$ breast cancer patients: results from the GEICAM/9906 trial. Breast Cancer Res 2014, 16:R38.

2. Filipits M, Rudas M, Jakesz R, Dubsky P, Fitzal F, Singer CF, Dietze O, Greil R, Jelen A, Sevelda P, Freibauer C, Müller V, Jänicke F, Schmidt M, Kölbl H, Rody A, Kaufmann M, Schroth W, Brauch H, Schwab M, Fritz P, Weber KE, Feder IS, Hennig G, Kronenwett R, Gehrmann M, Gnant M, EP Investigators: A new molecular predictor of distant recurrence in ER-positive, HER2negative breast cancer adds independent information to conventional clinical risk factors. Clin Cancer Res 2011, 17:6012-6020.

3. Simon RM, Paik S, Hayes DF: Use of archived specimens in evaluation of prognostic and predictive biomarkers. J Natl Cancer Inst 2009, 101:1446-1452. 
4. Dubsky P, Brase JC, Jakesz R, Rudas M, Singer CF, Greil R, Dietze O, Luisser I, Klug E, Sedivy R, Bachner M, Mayr D, Schmidt M, Gehrmann MC, Petry C, Weber KE, Fisch K, Kronenwett R, Gnant M, Filipits M, Austrian Breast and Colorectal Cancer Study Group (ABCSG): The EndoPredict score provides prognostic information on late distant metastases in $\mathrm{ER}^{+} / \mathrm{HER}^{-}$breast cancer patients. Br J Cancer 2013, 109:2959-2964.

5. Gray RG, Rea D, Handley K, Bowden SJ, Perry P, Earl HM, Poole CJ, Bates T, Chetiyawardana S, Dewar JA, Fernando IN, Grieve R, Nicoll J, Rayter Z, Robinson A, Salman A, Yarnold J, Bathers S, Marshall A, Lee M, aTTom Collaborative Group: aTTom: long-term effects of continuing adjuvant tamoxifen to 10 years versus stopping at 5 years in 6,953 women with early breast cancer. J Clin Oncol 2013, 31:5.

6. Davies C, Pan H, Godwin J, Gray R, Arriagada R, Raina V, Abraham M, Medeiros Alencar VH, Badran A, Bonfill X, Bradbury J, Clarke M, Collins R, Davis SR, Delmestri A, Forbes JF, Haddad P, Hou MF, Inbar M, Khaled H, Kielanowska J, Kwan WH, Mathew BS, Mittra I, Müller B, Nicolucci A, Peralta O, Pernas F, Petruzelka L, Pienkowski T, et al: Adjuvant Tamoxifen: Longer Against Shorter (ATLAS) Collaborative Group. Long-term effects of continuing adjuvant tamoxifen to 10 years versus stopping at 5 years after diagnosis of oestrogen receptor-positive breast cancer: ATLAS, a randomised trial. Lancet 2013, 381:805-816.

7. Ioannidis JP: Is molecular profiling ready for use in clinical decision making? Oncologist 2007, 12:301-311.

8. Vant Veer $L$, Dai H, van de Vijver MJ, He YD, Hart AA, Mao M, Peterse HL, van der Kooy K, Marton MJ, Witteveen AT, Schreiber GJ, Kerkhoven RM, Roberts C, Linsley PS, Bernards R, Friend SH: Gene expression profiling predicts clinical outcome of breast cancer. Nature 2002, 415:530-536.

9. Paik S, Shak S, Tang G, Kim C, Baker J, Cronin M, Baehner FL, Walker MG, Watson D, Park T, Hiller W, Fisher ER, Wickerham DL, Bryant J, Wolmark N: A multigene assay to predict recurrence of tamoxifen-treated, node-negative breast cancer. N Engl J Med 2004, 351:2817-2826.

10. Sestak I, Dowsett M, Zabaglo L, Lopez-Knowles E, Ferree S, Cowens JW, Cuzick J: Factors predicting late recurrence for estrogen receptor-positive breast cancer. J Natl Cancer Inst 2013, 105:1504-1511.

11. Ma XJ, Salunga R, Dahiya S, Wang W, Carney E, Durbecq V, Harris A, Goss P, Sotiriou C, Erlander M, Sgroi D: A five-gene molecular grade index and HOXB13:IL17BR are complementary prognostic factors in early stage breast cancer. Clin Cancer Res 2008, 14:2601-2608.

12. Parker JS, Mullins $M$, Cheang MC, Leung S, Voduc D, Vickery T, Davies S, Fauron C, He X, Hu Z, Quackenbush JF, Stijleman IJ, Palazzo J, Marron JS, Nobel AB, Mardis E, Nielsen TO, Ellis MJ, Perou CM, Bernard PS: Supervised risk predictor of breast cancer based on intrinsic subtypes. J Clin Oncol 2009, 27:1160-1167.

13. Sgroi DC, Sestak I, Cuzick J, Zhang Y, Schnabel CA, Schroeder B, Erlander MG, Dunbier A, Sidhu K, Lopez-Knowles E, Goss PE, Dowsett M: Prediction of late distant recurrence in patients with oestrogen-receptor-positive breast cancer: a prospective comparison of the breast-cancer index $(\mathrm{BCl})$ assay, 21-gene recurrence score, and IHC4 in the TransATAC study population. Lancet Oncol 2013, 14:1067-1076.

14. Cuzick J, Dowsett M, Pineda S, Wale C, Salter J, Quinn E, Zabaglo L, Mallon E, Green AR, Ellis 1O, Howell A, Buzdar AU, Forbes JF: Prognostic value of a combined estrogen receptor, progesterone receptor, $\mathrm{Ki}-67$, and human epidermal growth factor receptor 2 immunohistochemical score and comparison with the Genomic Health recurrence score in early breast cancer. J Clin Oncol 2011, 29:4273-4278.

15. Ross DT, Kim CY, Tang G, Bohn OL, Beck RA, Ring BZ, Seitz RS, Paik S, Costantino JP, Wolmark N: Chemosensitivity and stratification by a five monoclonal antibody immunohistochemistry test in the NSABP B14 and B20 trials. Clin Cancer Res 2008, 14:6602-6609.

16. Polley MY, Leung SC, MCShane LM, Gao D, Hugh JC, Mastropasqua MG, Viale G, Zabaglo LA, Penault-Llorca F, Bartlett JM, Gown AM, Symmans WF, Piper T, Mehl E, Enos RA, Hayes DF, Dowsett M, Nielsen TO, International Ki67 in Breast Cancer Working Group of the Breast International Group and North American Breast Cancer Group: An international Ki67 reproducibility study. J Natl Cancer Inst 2013, 105:1897-1906.

17. Zhang $Y$, Schnabel CA, Schroeder BE, Jerevall PL, Jankowitz RC, Fornander T, Stål O, Brufsky AM, Sgroi D, Erlander MG: Breast cancer index identifies early-stage estrogen receptor-positive breast cancer patients at risk for early- and late-distant recurrence. Clin Cancer Res 2013, 19:4196-4205.
18. Denkert C, Kronenwett R, Schlake W, Bohmann K, Penzel R, Weber KE, Höfler H, Lehmann U, Schirmacher P, Specht K, Rudas M, Kreipe HH, Schraml P, Schlake G, Bago-Horvath Z, Tiecke F, Varga Z, Moch H, Schmidt M, Prinzler J, Kerjaschki D, Sinn BV, Müller BM, Filipits M, Petry C, Dietel M: Decentral gene expression analysis for ER+/Her2- breast cancer: results of a proficiency testing program for the EndoPredict assay. Virchows Arch 2012, 460:251-259.

19. Kronenwett R, Bohmann K, Prinzler J, Sinn BV, Haufe F, Roth C, Averdick M, Ropers T, Windbergs C, Brase JC, Weber KE, Fisch K, Müller BM, Schmidt M, Filipits M, Dubsky P, Petry C, Dietel M, Denkert C: Decentral gene expression analysis: analytical validation of the Endopredict genomic multianalyte breast cancer prognosis test. BMC Cancer 2012, 12:456.

20. Christiansen J, Bartlett JMS, Gustavson M, Rimm D, Robson T, Van De Velde CJH, Hasenburg A, Kieback DG, Putter H, Markopoulos C, Dirix LY, Seynaeve CM, Rea D: Validation of IHC4 algorithms for prediction of risk of recurrence in early breast cancer using both conventional and quantitative IHC approaches. J Clin Oncol 2012, 30:517.

21. Cronin M, Sangli C, Liu ML, Pho M, Dutta D, Nguyen A, Jeong J, Wu J, Langone KC, Watson D: Analytical validation of the Oncotype DX genomic diagnostic test for recurrence prognosis and therapeutic response prediction in node-negative, estrogen receptor-positive breast cancer. Clin Chem 2007, 53:1084-1091.

22. Mamounas EP, Tang G, Paik S, Baehner FL, Liu Q, Jeong J-H, Kim S-R, Butler SM, Jamshidian F, Cherbavaz DB, Sing AP, Shak S, Julian TB, Lembersky BC, Wickerham DL, Costantino JP, Wolmark N: Association between the 21-gene recurrence score (RS) and benefit from adjuvant paclitaxel (Pac) in node-positive $(\mathrm{N}+)$, ER-positive breast cancer patients (pts): Results from NSABP B-28. Proc SABCS 2012, Abstract:S1-S10.

23. Albain KS, Barlow WE, Shak S, Hortobagyi GN, Livingston RB, Yeh IT, Ravdin P, Bugarini R, Baehner FL, Davidson NE, Sledge GW, Winer EP, Hudis C, Ingle JN, Perez EA, Pritchard Kl, Shepherd L, Gralow JR, Yoshizawa C, Allred DC, Osborne CK, Hayes DF, Breast Cancer Intergroup of North America: Prognostic and predictive value of the 21-gene recurrence score assay in postmenopausal women with node-positive, oestrogen-receptor-positive breast cancer on chemotherapy: a retrospective analysis of a randomised trial. Lancet Oncol 2010, 11:55-65.

24. Paik S, Shak S, Tang G, Kim C, Baker J, Cronin M, Watson D, Bryant J, Costantino J, Wolmark N: Expression of the 21 genes in the Recurrence Score assay and tamoxifen clinical benefit in the NSABP study B-14 of node negative, estrogen receptor positive breast cancer. J Clin Oncol 2005, 23:510.

25. Paik S, Tang G, Shak S, Kim C, Baker J, Kim W, Cronin M, Baehner FL, Watson D, Bryant J, Costantino JP, Geyer CE Jr, Wickerham DL, Wolmark N: Gene expression and benefit of chemotherapy in women with node-negative, estrogen receptor-positive breast cancer. J Clin Oncol 2006, 24:3726-3734.

26. Cheang MC, Voduc KD, Tu D, Jiang S, Leung S, Chia SK, Shepherd LE, Levine MN, Pritchard KI, Davies S, Stijleman IJ, Davis C, Ebbert MT, Parker JS, Ellis MJ, Bernard PS, Perou CM, Nielsen TO: Responsiveness of intrinsic subtypes to adjuvant anthracycline substitution in the NCIC.CTG MA.5 randomized trial. Clin Cancer Res 2012, 18:2402-2412

27. Chia SK, Bramwell VH, Tu D, Shepherd LE, Jiang S, Vickery T, Mardis E, Leung S, Ung K, Pritchard KI, Parker JS, Bernard PS, Perou CM, Ellis MJ, Nielsen TO: A 50-gene intrinsic subtype classifier for prognosis and prediction of benefit from adjuvant tamoxifen. Clin Cancer Res 2012, 18:4465-4472.

28. Bastien RR, Rodríguez-Lescure Á, Ebbert MT, Prat A, Munárriz B, Rowe L, Miller P, Ruiz-Borrego M, Anderson D, Lyons B, Álvarez I, Dowell T, Wall D, Seguí MÁ, Barley L, Boucher KM, Alba E, Pappas L, Davis CA, Aranda I, Fauron C, Stijleman IJ, Palacios J, Antón A, Carrasco E, Caballero R, Ellis MJ, Nielsen TO, Perou CM, Astill M, Bernard PS, Martín M: PAM50 breast cancer subtyping by RT-qPCR and concordance with standard clinical molecular markers. BMC Med Genomics 2012, 5:44.

29. Prat A, Bianchini G, Thomas M, Belousov A, Cheang MC, Koehler A, Gómez P, Semiglazov V, Eiermann W, Tjulandin S, Byakhow M, Bermejo B, Zambetti M, Vazquez F, Gianni L, Baselga J: Research-based PAM50 subtype predictor identifies higher responses and improved survival outcomes in HER2-positive breast cancer in the NOAH study. Clin Cancer Res 2014, 20:511-521.

30. Martín M, Prat A, Rodríguez-Lescure A, Caballero R, Ebbert MT, Munárriz B, Ruiz- Borrego M, Bastien RR, Crespo C, Davis C, Rodríguez CA, López-Vega JM, Furió V, García AM, Casas M, Ellis MJ, Berry DA, Pitcher BN, Harris L, Ruiz A, Winer E, Hudis C, Stijleman IJ, Tuck DP, Carrasco E, Perou CM, Bernard PS: PAM50 proliferation score as a predictor of weekly paclitaxel benefit in breast cancer. Breast Cancer Res Treat 2013, 138:457-466. 
31. Nielsen T, Wallden B, Schaper C, Ferree S, Liu S, Gao D, Barry G, Dowidar N, Maysuria M, Storhoff J: Analytical validation of the PAM50-based Prosigna Breast Cancer Prognostic Gene Signature Assay and nCounter Analysis System using formalin-fixed paraffin-embedded breast tumor specimens. BMC Cancer 2014, 14:177.

32. Dowsett M, Sestak I, Lopez-Knowles E, Sidhu K, Dunbier AK, Cowens JW, Ferree S, Storhoff J, Schaper C, Cuzick J: Comparison of PAM50 risk of recurrence score with oncotype $\mathrm{DX}$ and IHC4 for predicting risk of distant recurrence after endocrine therapy. J Clin Oncol 2013, 31:2783-2790.

33. Filipits M, Nielsen TO, Rudas M, Greil R, Stöger H, Jakesz R, Bago-Horvath Z, Dietze O, Regitnig P, Gruber-Rossipal C, Müller-Holzner E, Singer CF, Mlineritsch B, Dubsky P, Bauernhofer T, Hubalek M, Knauer M, Trapl H, Fesl C, Schaper C, Ferree S, Liu S, Cowens JW, Gnant M, Austrian Breast and Colorectal Cancer Study Group: The PAM50 risk-of-recurrence score predicts risk for late distant recurrence after endocrine therapy in postmenopausal women with endocrine-responsive early breast cancer. Clin Cancer Res 2014, 20:1298-1305.

$10.1186 / \mathrm{bcr} 3688$

Cite this article as: Kos and Nielsen: Developing a new generation of breast cancer clinical gene expression tests.

Breast Cancer Research 2014, 16:103 